Voix et Images

volxetimages

\title{
Forme et signification du temps et du discours immédiat dans \\ Poussière sur la ville : le récit d'une victoire
}

\section{Pierre Hébert}

Volume 2, numéro 2, décembre 1976

Paul Chamberland

URI : https://id.erudit.org/iderudit/200054ar

DOI : https://doi.org/10.7202/200054ar

Aller au sommaire du numéro

Éditeur(s)

Les Presses de l'Université du Québec

ISSN

0318-9201 (imprimé)

1705-933X (numérique)

Découvrir la revue

\section{Citer cet article}

Hébert, P. (1976). Forme et signification du temps et du discours immédiat dans Poussière sur la ville : le récit d'une victoire. Voix et Images, 2(2), 209-230.

https://doi.org/10.7202/200054ar d'utilisation que vous pouvez consulter en ligne. 


\section{Forme et signification du temps et du discours immédiat dans Poussière sur la ville: le récit d'une victoire}

Vous lisez une première étude sur Poussière sur la ville d'André Langevin: l'on vous parle de fatalité. Vous en lisez une seconde: l'on vous parle d'échec. Vous en inférez, avec raison, une relation de cause à effet: la fatalité rend l'échec nécessaire. Cela est bien connu...

Mais est-ce tout?

Êtes-vous mieux renseigné sur la forme et le fonctionnement du récit? Où s'enracine la signification que l'on vous propose? N'y aurait-il pas une autre façon de lire Poussière sur la ville?

Évidemment, nous répondons affirmativement à la dernière question, mais à la condition essentielle que l'analyse du roman s'articule dans une double lecture: d'abord une lecture du fonctionnement et de la forme du discours du récit, ensuite une lecture de la signification de cette même forme du discours narratif. En conséquence, nous avons l'intention de décrire le fonctionnement et la forme du récit Poussière sur la ville à deux de ses niveaux, le temps (ordre, durée et fréquence) et le discours immédiat (ou monologue intérieur) ${ }^{1}$. Les études du fonctionnement textuel romanesque ne sont pas légion, et encore moins celles qui prétendent arracher par ce biais une signification au texte. Avec une méthode cependant différente de la science, nous endosserons donc le projet de Rousset $^{2}$, soit d'enraciner la signification d'un récit dans sa forme.

\subsection{FORME ET SIGNIFICATION DU TEMPS}

Quid est ergo tempus? Si nemo ex me quaerat, scio; si quaerenti explicare velim, nescio.

Saint Augustin

Le poids même de l'évidence oblige tout scientifique, tout chercheur, bref tout Don Quichotte qui part en campagne contre le Temps à bien spécifier, à l'heure même des projets, que les résultats seront inversement 
proportionnels aux efforts et que le Sphinx gardera son secret. Ainsi Butor valide-t-il le passage-cliché de saint Augustin que nous avons mis en exergue: «[...] les structures chronologiques de fait sont d'une complexité tellement vertigineuse que les schémas les plus ingénieux utilisés soit dans l'élaboration de l'ouvrage, soit dans son exploration critique, ne pourront jamais être que de grossières approximations ${ }^{3}$. "

Irrespectueuses pour les proverbes, les prochaines lignes poursuivront deux lièvres à la fois. Elles oscilleront entre la "poétique", comme connaissance théorique du récit littéraire (de ses conditions d'existence), et le texte particulier de Poussière sur la ville, afin de connaître la forme spécifique de son discours-occurrence. Dans le premier temps, théorique, nous proposons une réflexion sur la nécessité temporelle du récit à deux niveaux, anthropologique et linguistique; une fois le fondement temporel du récit assuré, nous pourrons alors, dans un deuxième temps, procéder à l'analyse critique de Poussière sur la ville à l'aide des concepts et méthodes élaborés par Gérard Genette dans Figures III, que nous considérons connus du lecteur ${ }^{4}$.

Notre ambition sera donc de nous écarter des approches classiques du temps (psychanalytiques, philosophiques et autres), et de considérer cette instance d'analyse comme forme romanesque, nous dégageant par là d'une vision myope de Poussière pour en repérer l'organisation temporelle spécifique.

\subsection{Les fondements temporels du récit}

D'une part, Claude Bremond affirme: “[...] la sémiologie du récit tire sa possibilité et sa fécondité de son enracinement dans une anthropologie ${ }^{5}$. D'autre part, l'analyse structurale et la sémiologie littéraire proclament le récit production linguistique et, de cette hypothèse, élaborent leur méthode et l'appuient dans ses fondements sur la science de la langue. La recherche du fondement temporel d'un récit doit donc s'organiser en deux temps: anthropologique et linguistique.

\subsection{Le fondement anthropologique}

Tout récit est réductible au modèle triadique que nous propose Claude Bremond, modèle arc-bouté sur les notions de rôles et de séquences ${ }^{6}$. Or il semble que si les fonctions ou les rôles se groupent en triades, une telle organisation manifeste une suite non seulement logique mais temporelle, et la réflexion en ce sens ne nous semble pas jusqu'à présent assez élaborée. Ainsi, s'il est vrai que le fondement d'une "géno-diégèse" se trouve dans la logique de ses possibles narratifs, et s'il est vrai également que cette logique séquentielle est non seulement historique mais aussi temporelle, il s'ensuivra que le temps est une forme à priori du récit littéraire au même' titre que les arcanes de sa logique narrative. 
Prenons le modèle de Bremond dans son sens le plus large, c'està-dire comme "succession d'évenements d'interèts humains dans l'unité d'une même action" ", pour lui donner, à ce stade-ci, un fondement temporel anthropologique. Il faudrait se rendre compte combien ce modèle, en s'amorçant par un acte à accomplir (au sens large) désigne la base même de l'activité humaine proprement dite. En effet, I'histoire de l'humanité, comme récit, comme accomplissement, a commencé avec la rupture de l'équilibre conduite-instinct: cette conduite instinctuelle, conduite sûre et sans hésitation parce qu'absolue, manifestée symboliquement par l'Éden, a cédé sa place à une nouvelle forme de conduite, celle de la raison, en rupture avec l'univers, et ce décollement, cette disjonction a marqué le début de l'humanité comme progression, comme tâche à accomplir: on pense à Hegel ou à Teilhard de Chardin.

En même temps que ce mouvement de création/destruction surgit une nouvelle donnée qui lui est inséparable: le temps. La conscience du temps comme accomplissement ou destruction de l'activité humaine est corollaire au mouvement décrit plus haut: quand il y a projet humain, il y a du même coup mort du présent perpétuel et début de l'Histoire. Prenons le modèle de Bremond dans son sens le plus large, pour «l'appliquer " à l'humanité; et cela se peut dans la mesure où il sera bien clair que ce modèle ne s'applique pas au récit seulement, mais à toute activité humaine: pour parler en termes hjemsleviens, le modèle de Bremond est la substance du contenu de tout récit et, à ce titre, il n'est pas seulement littéraire.

Ce bref excursus ne vise qu'un but: montrer comment - par analogie - un récit, quel qu'il soit, commence par la rupture d'un équilibre, que le manque est origine de l'histoire (humaine ou littéraire), et que cette rupture inaugure une démarche non seulement logique mais temporelle. Le récit, avant d'être littéraire, est humain. De même l'est son temps. Le comportement humain est un récit temporel et de la même manière le récit humain est un comportement temporel.

\subsection{Le fondement linguistique}

Ce n'est pas par errance, par mégarde ou par vice de méthode que nous venons de "remonter au déluge" pour parler de Bremond: c'est que son modèle, appliqué au comportement humain, s'avère très éclairant et solidement fondé.

Restreignons maintenant notre champ focal, et cessons de considérer les choses par le gros bout de la lorgnette. Appliqué au récit, le modèle de Bremond trouve facilement une conversion linguistique si on admet qu'une action (=fonction) puisse, comme le dit Todorov, se résumer par une phrase, et qu'à chaque triade (=séquence) correspondent trois temps verbaux principaux, les trois seuls temps qui, d'ailleurs, se retrouvent à tous les modes: futur, présent, passé composé. Ainsi les trois séquences de Bremond seraient désignées analogiquement par ces trois verbes: 
1. Fonction qui ouvre: futur, type Je/tu/il fera quelque chose;

2. Fonction qui réalise : présent, type Je/tu/il fait;

3. Fonction qui clôt: passé composé (parfait de présent), type Je/tu/il a fait.

Ce dernier type, manifesté par un temps composé, peut paraître douteux, voire gratuit; mais il est validé par l'affirmation catégorique de Benveniste: le parfait «consiste à présenter la notion comme 'accomplie' par rapport au moment considéré, et la situation 'actuelle' résultant de cet accomplissement temporalisé ${ }^{8}$ ». Admirons cette fin de phrase: «accomplissement temporalisé». Causalité et temporalité s'échangent des répliques dans le cinétisme que leur confère le modèle de Bremond ${ }^{9}$. Le temps du récit existe donc non seulement au niveau d'accident de l'histoire, c'està-dire en tant que discours, mais surtout au niveau même du récit considéré comme grand texte théorique.

Notre problème essentiel, dans l'étude du temps de Poussière, n'est donc pas tellement de trouver une unité de mesure, mais de découvrir sur quel objet s'exercera ladite unité. A travers le foisonnement événementiel, notre étude se limitera à la macro-structure, étant bien entendu qu'une macro-structure est, par rapport aux séquences de Bremond, un super-signe ${ }^{10}$, ce qui n'infirme en rien le fondement temporel élaboré au cours des dernières pages. Ainsi, étudier la temporalité de Poussière sur la ville, ce sera mesurer selon l'ordre, la durée et la fréquence des séquences pertinentes, au niveau macroscopique.

\subsection{Le fonctionnement temporel: l'ordre (les analepses)}

Le premier aspect à étudier est donc celui de l'ordre. Mais supposons établie auparavant une solide chronologie interne du roman, où le temps marqué 0 sera la coïncidence $T H=T N^{11}$, et où les événements antérieurs seront marqués $-1,-2$, etc. (ex.: avertissement de Kouri, -1), et les événements ultérieurs 1, 2, etc. (ex.: le déjeuner du lendemain 20 déc., 1). Cette chronologie sera notre repère de l'avant et de l'après, où le temps 0 sera la frontière entre ce qui est interne et ce qui est externe. Nous pouvons alors commencer une étude de l'ordre ${ }^{12}$.

Bien sûr, cette étude est possible dans la mesure où l'on veut bien admettre que le récit a deux temps: celui de son histoire et celui de sa narration. Ainsi le temps de la narration, nécessairement linéaire, peut prendre en charge des événements déplacés de leurs positions chronologiques successives. Si l'on représente, comme le fait Genette, par une lettre le TN, et par un chiffre la position chronologique du segment historique tel que décrit plus haut, on pourrait avoir, hypothétiquement:

\section{A5 B4 C3 D2 E1}

c'est-à-dire une récupération progressive par la narration des événements antérieurs à celle-ci. 
Dans Poussière, nous pouvons mettre en exergue huit analepses macroscopiques. Voici leur contenu et leur position temporelle:

Analepses

Pages

$12,13,15,19,23,38-1$

1. Avertissements de Kouri

2. Arrivée à MacKlin

3. Au restaurant et au cinéma

4. Au déjeuner

5. Première "possession"

6. Avec Hétu

7. Récit de la mort

8. Journée de travail

$\begin{array}{cl}20 & -3 \\ 25 & -2 \\ 130-131 & +10 \\ 146-148 & -4 \\ 169-173 & 14 \text { (plus ou moins) } \\ 192 & 17 \\ 205-211 & 19\end{array}$

Du point de vue de leur portée, ces analepses présentent le-mouvement suivant:

1. externe

2. externe

3. externe

4. interne

5. externe

6. interne

7. interne

8. interne

Ce passage de l'externe à l'interne est sans doute dû au type d'amorce du récit “in medias res »: ce genre de commencement oblige le récit à récupérer certains antécédents de sa fable qui nous sont par définition étrangers. Les quatre analepses externes permettent une extension remarquable du champ temporel du récit (mi-juillet, c'est-à-dire plus de quatre mois). Ce qu'il importe de remarquer, c'est combien le contenu même de l'histoire motive le recours à de nombreuses analepses: "La quiétude de la maison donne du relief à des moments du passé, m'aide à leur prêter un sens nouveau ${ }^{13}$." ( $P$., p. 19) Nous touchons là la fonction canonique de l'analepse:

[...] venir modifier après coup la signification des événements passés, soit en rendant signifiant ce qui ne l'était pas, soit en réfutant une première interprétation et en la remplaçant par une nouvelle ${ }^{14}$.

“Ce procédé est évidemment l'un des moyens les plus efficaces de la circulation du sens dans le roman ${ }^{15}$." L'analepse la plus remarquable, la seule externe qui soit complète, est sans l'ombre d'un doute l'avertissement de Kouri. Quand le roman débute, cet avertissement a déjà eu lieu; il nous parvient par la mémoire d'Alain, d'abord une première fois (analepse complétive) puis de façon répétée. On assiste, outre le piétinement historique mis en évidence lors du chapitre précédent, à une multiplication des instan- 
ces mémorielles du début; ce phénomène, qui nous conduit prématurément dans l'univers de la fréquence, retient l'attention quant à sa valeur dans le dynamisme même de l'œuvre. C'est en effet à cause de l'avertissement de Kouri que le récit (comme rupture) a lieu; de cet avertissement, pourtant, nous n'avons jamais été témoins, et Alain devient la patrie même de sa propre désintégration aux yeux du lecteur. Le personnage prend en charge toute la fable du récit, il est vraiment un assimilateur de données, il fait ce premier apprentissage de la vérité. Ce qui faisait la particularité de la Recherche de Proust se répète à nouveau: «[...] la multiplication des instances mémorielles, et par suite la multiplication des débuts ${ }^{16} \%$. Le roman vit donc, encore à la manière du récit proustien, d'une dialectique séquentielle entre l'éveil rétrospectif et l'innocence du moment présent, d'un jeu de répliques entre “Qu'a-t-il voulu dire le Syrien ? " d'une part, et «De mon émoi de la veille il ne subsiste rien" d'autre part. (P., p. 13 et 43)

Les autres analepses, tant du point de vue de leur portée que de leur amplitude, ne présentent rien de plus que les considérations générales que nous en avons tirées au début.

Nous remarquons également un mouvement du complexe au simple. En effet, les vingt-neuf premières pages comportent trois retours (Kouri -6 fois -, arrivée à Macklin, au restaurant et au cinéma), lesquels couvrent environ dix-huit pages. Cette proportion, à une échelle aussi grande, ne se retrouvera nulle part ailleurs dans le roman. Les quatre analepses finales seront très espacées dans la topologie du roman, et auront une portée infime, comme dans le cas des deux dernières.

\subsection{Le fonctionnement temporel: l'ordre (les prolepses)}

Plus courtes sont les prolepses (de fait, elles se résument souvent à une ligne ou deux). Voici l'énumération des plus importantes:

\section{Prolepses}

1. Agissements de Madeleine

2. Sens de leur vie

3. Sens de leur vie

4. Décision d'Alain

5. Décision d'Alain

6. Agissements de Madeleine

7. Propositions d'Alain

8. Décision d'Alain
Pages

Positions chronologiques

68
73
130
$152-153$
174
176
187
213

(Aucune position précision.) identifiable avec

II est tout de même assez étonnant de constater que ces prolepses ne peuvent, en aucun cas, se greffer avec précision sur une échelle chrono- 
logique. Et pourtant cela n'interdit pas à ces distorsions temporelles de jouer un rôle prémonitoire: en quelques occasions, les prolepses nous préparent à la fin tragique de Madeleine:

Je la vois morte sans avoir été heureuse, morte désespérée parce qu'elle n'étreindra jamais ce qui l'aurait comblée, morte toute seule avec son ardeur inassouvie, son petit corps contracté dans un dernier spasme de fierté. Que m'importera alors d'avoir été trahi: je ne la revendiquerai plus pour femme. ( $P$., p. 152)

Cette «ardeur inassouvie» qui anime Madeleine et qui la plongera (selon Alain) dans la mort est décrite par ce dernier en vertu du filtre fataliste de la prolepse:

Elle recommencera demain. Ce coup-ci elle montre de la ténacité. Elle est décidée à en finir, à voir au fond des choses une fois pour toutes. [...] Elle ira au fond des choses, forcera la réalité à épouser son rêve aussi longtemps qu'elle le pourra. ( $P$., p. 176-177)

Nous pouvons constater dans le récit, cette fois, un mouvement du simple au complexe: plus on avance dans le récit, plus les prolepses se font nombreuses.

\subsection{Le fonctionnement temporel: la durée}

[...] l'isochronisme d'un récit peut aussi se définir, comme celui d'une pendule par exemple, non plus relativement, par comparaison entre sa durée et celle de l'histoire qu'il raconte, mais de manière en quelque sorte absolue et autonome, comme constance de vitesse ${ }^{17}$.

Cette constance de vitesse résultera de la mise en rapport entre la durée de l'histoire (dans la mesure où celle-ci est quantifiable) et la longueur du texte qui subsume la séquence historique. Le but de cette étude de durée sera, par conséquent, d'évaluer l'isochronie ou, ce qui revient au même, l'anisochronie éventuelle d'un récit ou encore, si l'on préfère, d'en évaluer le rythme, stable, accéléré ou désaccéléré.

La méthode ne semble encore valable qu'au niveau macroscopique: quantifier la durée diégétique des grandes articulations narratives est déjà une tâche approximative et périlleuse en soi, sans au surplus atomiser le texte, au risque assuré de se retrouver devant l'incapacité de mesurer la durée de l'histoire.

Dans Poussière, le travail consistera donc d'une part à découper les articulations narratives macroscopiques, puis à évaluer la durée temporelle de chacune des articulations. Mais cette tâche s'accomplit sous le signe d'une double approximation. Approximation d'abord quant au découpage lui-même: le critère de division des articulations sera une "rupture temporelle et/ou spatiale importante ${ }^{18}$ ", étant bien entendu que c'est à défaut d'instrument plus tranchant que le critère démarcatif résultera d'une rupture «importante», donc très relative; approximation, en second lieu, quant à la quantification de la durée diégétique du segment déjà arbitrai- 
rement découpé: souventes fois cette durée ne s'acquiert que par une évaluation connue par déduction.

Ces lacunes admises, on peut présenter le découpage de Poussière en douze grandes séquences; regardant tour à tour:

a. leur teneur événementielle;

b. leur position chronologique;

c. leur importance textuelle (nombre de pages);

d. leur durée diégétique;

e. le cas échéant, leurs caractères spécifiques.

\section{Séquence 1:}

a. L'avertissement de Kouri, Alain dehors puis chez lui;

b. le jeudi 19 décembre;

c. pages 11 à 39,29 pages ;

d. la durée de cette séquence est difficile à évaluer: il est presque minuit (p. 11), l'avertissement a eu lieu, Alain est dehors, puis rentre chez lui, réfléchit et s'endort. On peut évaluer le temps à environ une heure;

e. il faut rappeler, bien sûr, l'importance des analepses dans cette première séquence.

\section{Séquence 2:}

a. Le déjeuner, à l'hôpital avec Lafleur, rencontres avec Prévost et «la grosse femme», puis au restaurant, chez le bijoutier; à la maison, chez la cardiaque, et retour à la maison;

b. le vendredi 20 décembre;

c. pages 41 à 77,36 pages;

d. supposons 8 heures, pour le déjeuner; à 17 heures Alain va au restaurant (p. 59), rentre chez lui à $18 \mathrm{~h} 30$ heures; Madeleine reste au cinéma au moins une heure et demie: supposons 20 heures; Madeleine revient, mais Alain reçoit un appel de la cardiaque, où il ne restera qu'à peine une heure (p. 83): au plus tard 22 heures, retour à la maison: un total de quatorze heures.

\section{Séquence 3:}

a. En taxi, à la maison, chez Kouri, puis à la maison;

b. le samedi 21 décembre;

c. pages 79 à 91,13 pages;

d. en taxi le matin, probablement vers 8 heures; le soir, à la maison, probablement vers 20 heures, après dîner: un total de douze heures.

\section{Séquence 4:}

a. En auto, à la maison, à l'hôtel, puis l'histoire de l'hydrocéphale;

b. le dimanche 22 décembre, et nuit du 23 ;

c. pages 95 à 121, 27 pages; 
d. en auto l'après-midi, et retour à 5 heures le 23 (p. 121): quatorze heures environ.

\section{Séquence 5 :}

a. A l'hôpital, puis avec Prévost;

b. le lundi 23 décembre;

c. pages $123-133,11$ pages;

d. à l'hôpital à 8 heures (p. 123), chez Prévost vers la fin de l'après-midi: un total de huit heures environ.

\section{Séquence 6:}

a. A la maison, la veille et la nuit de Noël;

b. le mardi 24 décembre, et la nuit du 25 ;

c. pages 135 à 156, 21 pages ;

d. vers 17 heures («demi-jour», p. 135) jusque vers 1 heure, après la messe de minuit: un total d'environ huit heures.

\section{Séquence 7 :}

a. Avec le curé;

b. début de février;

c. pages 159-165, 7 pages ;

d. on peut supposer une heure au maximum.

\section{Séquence 8:}

a. A la maison, réunion Alain, Madeleine et Richard Hétu;

b. un samedi de février;

c. pages $166-177,11$ pages;

d. évaluation de cette séquence: une heure;

e. cette séquence comporte deux analepses, dont la seconde présente un sommaire fort intéressant, qui a pour but de combler le vide entre la nuit de Noël et ce samedi de février:

Madeleine s'est transformée dès le lendemain de Noël, renforcie peutêtre par mon renoncement. Elle s'est mise à vivre son amour au grand jour, traînant à sa suite un Richard hébété, paraissant toujours marcher sur des charbons ardents. (P., p. 170)

\section{Séquence 9:}

a. À la maison, au dîner; Madeleine va au cinéma;

b. le jeudi suivant (p. 180);

c. pages 179 à 183,5 pages ;

d. de la fin de l'après-midi au début de la soirée, environ trois heures.

Séquence 10:

a. Départ de Madeleine, sa mort, retour à la maison; 
b. le lendemain, vendredi;

c. pages 185-197, 12 pages;

d. de sept heures du soir (p. 185), jusqu'à environ deux heures plus tard.

\section{Séquence 11:}

a. Après la mort;

b. aucune indication;

c. pages 199 à 201, 3 pages;

d. quelques minutes?;

e. en réalité, cette séquence est un sommaire très important, qui reprend les différents événements depuis la mort de Madeleine jusqu'à ce moment: enquête, Thérèse, etc.

\section{Séquence 12:}

a. La décision d'Alain;

b. trois mois après la mort;

c. pages 203 à 213,11 pages;

d. au maximum une demi-heure;

e. vue la grande distance entre cette séquence et la précédente, on retrouve un sommaire qui veut aussi combler le vide: page 205.

Afin de rendre le rythme du récit beaucoup plus visible, nous proposons une synthèse des pages précédentes dans le tableau suivant:

$\begin{array}{rrrr}\text { Séquences } & \text { Pages } & \text { Duree } & \text { Proportion (arrondie) } \\ 1 & & & \\ 2 & 29 & 1 \text { heure } & 1 \text { page: } 2 \text { minutes } \\ 3 & 36 & 14 \text { heures } & 1 \text { page: } 25 \text { minutes } \\ 4 & 13 & 12 \text { heures } & 1 \text { page: } 60 \text { minutes } \\ 5 & 27 & 14 \text { heures } & 1 \text { page: } 20 \text { minutes } \\ 6 & 11 & 8 \text { heures } & 1 \text { page: } 42 \text { minutes } \\ 7 & 21 & 8 \text { heures } & 1 \text { page: } 24 \text { minutes } \\ 8 & 7 & 1 \text { heure } & 1 \text { page: } 9 \text { minutes } \\ 9 & 11 & 1 \text { heure } & 1 \text { page: } 5 \text { minutes } \\ 10 & 5 & 3 \text { heures } & 1 \text { page: } 36 \text { minutes } \\ 11 & 12 & 2 \text { heures } & 1 \text { page: } 10 \text { minutes } \\ 12 & 3 & 5 \text { minutes } & 1 \text { page: } 1.6 \text { minutes } \\ & 11 & 30 \text { minutes } & 1 \text { page: } 2.8 \text { minutes }\end{array}$


Et ainsi, la courbe du récit prendrait cette allure ${ }^{\star}$ :

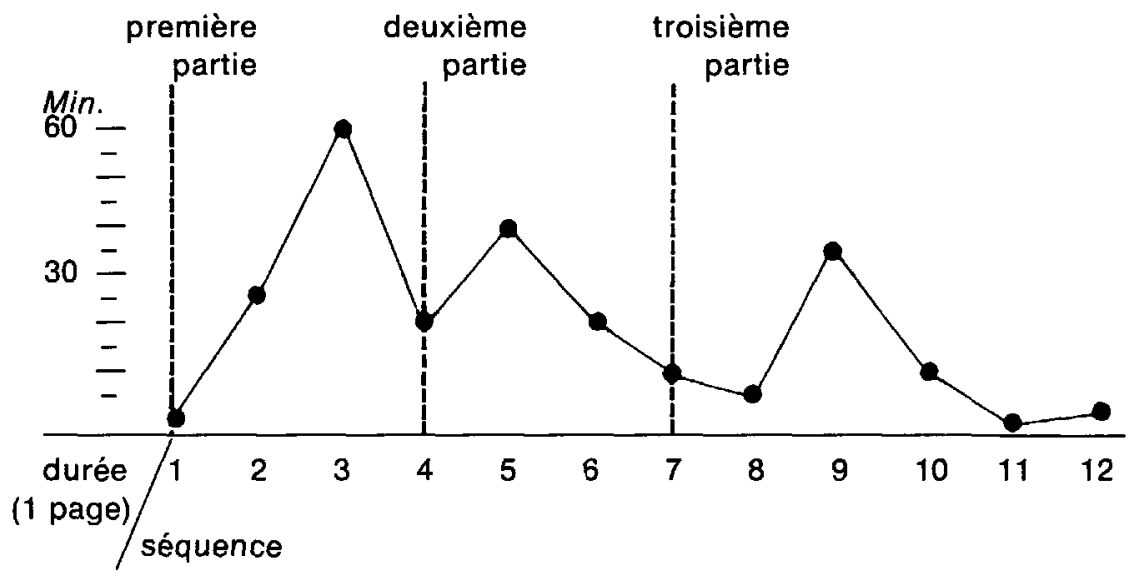

- Ce tableau doit être lu ainsi : horizontalement, les chiffres 1 à 12 désignent les 12 séquences découpées pour l'étude de la durée; verticalement, le tableau est gradué en minutes. En conséquence, chaque séquence se voit attribuer un point dans le tableau qui rende compte de sa durée en nombre de minutes par page. Dans le cas de la séquence 1, par exemple, dont le rapport est 1 page: 2 minutes, on placera un point dans le tableau au-dessus du chiffre 1, et vis-à-vis, quant à la graduation, de ce qui équivaut à 2 minutes. Tous les points sont ensuite reliés entre eux par un trait continu.

Il est assez étonnant de constater comment chacune des parties du roman suit une courbe similaire:

première partie:

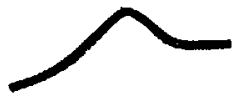

deuxième partie :

troisième partie :

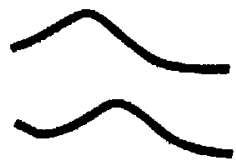

et que le récit lui-même suit une courbe analogue à celle des trois parties:

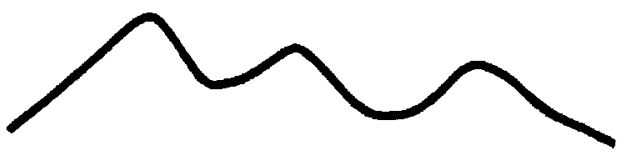

dans la mesure où précisément le début et la fin sont plus abaissés que le corps même du récit.

Ainsi il est permis d'affirmer à nouveau, cette fois par le biais du rythme du récit, que l'origine et la fin de ce dernier présentent un ralentis- 
sement remarquable et que, lorsque nous chercherons une signification aux formes du texte, il nous faudra examiner particulièrement cette origine et cette fin du récit, comme si ces deux extrémités pouvaient avoir des propriétés spécifiques: on pourra voir qu'elles sont surcodées, c'est-à-dire qu'elles contiennent une signification spécifique par rapport au corps du texte.

\subsection{Le fonctionnement temporel: la fréquence}

Poussière, dans son départ historique, réalise le type parfait du récit répétitif, qui peut se schématiser par $n R / 1 \mathrm{H}^{19}$. On sait que le récit s'ouvre sur une analepse: l'avertissement de Kouri. Cet avertissement est à l'origine de tout, et pourtant nous n'en avons jamais été témoins. Mais, et c'est là l'intérêt de ce phénomène, Alain ne fait pas que rappeler cet événement, il le répète, de telle sorte que le récit est constitué de séquences ouvertes par la répétition de ce même événement passé. L'histoire du récit, à ce niveau, n'est rien d'autre que la narration plurielle d'un événement unique amorçant des séquences de dégradation. Ce type de fréquence n'appartient peut-être qu'à un récit où le narrateur est soit autodiégétique, soit en focalisation zéro, c'est-à-dire omniscient.

La fréquence, dans Poussière, est donc manifestée par la réitération dans la narration d'un même événement historique, l'avertissement de Kouri. Dans l'organisation séquentielle que nous donnons plus bas, notons que l'avertissement de Kouri est répété huit fois, correspondant aux huit premières séquences. Signalons que succède alors à l'avertissement, comme point de départ séquentiel, soit l'avertissement de Jim, répété trois fois, soit la présence de Kouri dans l'esprit d'Alain, répété cinq fois. On pourra remarquer, sans aller plus loin dans cette constatation, que toutes les séquences sont identiques dans leur mouvement triptyque:

1. éveil, par les avertissements ou la présence en pensée de Kouri;

2. conscience, réflexion sur l'agir de Madeleine;

3. tentative de fuite, par un refus de la conscience, le tout, bien sûr, dans la perspective d'Alain.

Voici comment peut s'articuler cette structure séquentielle à valeur essentiellement répétitive:

éveil: $\quad$ Kouri et son premier avertissement. (p. 11)

1. conscience: Alain sort du restaurant et, angoissé, regarde sa maison. (11)

tentative: «Je quittai le restaurant sans mot dire [...]»(12)

éveil: $\quad$ Alain repense à l'avertissement de Kouri. (12)

2. conscience: Alain soupçonne Kouri d'avoir "levé le rideau». (13)

tentative: Fuite d'Alain: "Je n'ai ni le goût, ni le temps de ces sortes de jeux. " (13) 
éveil: "Qu'a voulu dire le Syrien?" (13)

3. conscience: Alain songe à Madeleine, souvent seule chez Kouri. (13) tentative: «Cela ne concerne que Madeleine et moi.» (13)

éveil: "Kouri a certainemement voulu dire davantage." (13)

4. conscience: Se présente sous forme de mémoire: Alain songe à Madeleine au restaurant. (14)

tentative: "Bah! le Syrien est assez simple d'esprit [...]": Alain fuit la conscience. (14)

éveil: $\quad$ Cinquième apparition de l'avertissement. (15)

5. conscience: Alain se rend compte qu'une part de la vie de Madeleine lui échappe. (15-19)

tentative: «A vrai dire, je ne songe guère à cela [...]" (19)

éveil: $\quad$ Sixième rappel de l'avertissement. (19)

6. conscience: Conscience par la mémoire. (21-23)

tentative: "J'avais oublié les paroles du Syrien. " (23)

éveil: $\quad$ Alain pense une septième fois à l'avertissement. (24) .

7. conscience: "[...] je m'abandonne à leur mouvement [...]" (23)

tentative: “J'essaie de penser à mon travail de demain. » (25)

éveil: $\quad$ Point de départ: huitième apparition de l'avertissement. (38)

8. conscience: Sous forme de mémoire par Alain, le lendemain de leur arrivée. (25)

tentative: «Tout s'embrouille et je m'endors un peu crispé [...]» (39)

éveil: $\quad$ Alain songe à Kouri. (43)

9. conscience: II interroge Thérèse, qui ravive son inquiétude. (43-44)

tentative: «Je me hâte [...] pour échapper à mes pensées. 》 (44)

éveil: $\quad$ Premier avertissement de Jim. (46)

10. conscience: "[...] je me fige automatiquement." (46)

tentative: Alain cherche "le moyen de l'empêcher de [lui] parler. " (46)'

éveil: $\quad$ Présence d'Alain chez Kouri (60); Kouri lui montre Madeleine qui est là. (61)

11. conscience: Alain songe à Madeleine. (63-64)

tentative: Alain veut fuir: "Je suis las [...]"; "N'en parlons plus." (66)

éveil: $\quad$ Présence d'Alain devant le restaurant de Kouri, ne pouvant se "résoudre à l'équivoque". (67)

12. conscience: Madeleine prend conscience d'une foule de sentiments. (68-69)

tentative: Achète un cadeau à Madeleine, pour "l'empêcher de pleurer». (70) 
éveil: $\quad$ Alain imagine Madeleine chez Kouri. (72)

13. conscience: "I "évoque le visage inquiétant de Madeleine». (72)

tentative: Du nouveau: Alain songe à accepter la conscience.

éveil: $\quad$ Madeleine est vue devant chez Kouri. (74)

14. conscience: Alain observe Madeleine dehors et chez lui. (74-75)

fuite: Un appel rompt cette rencontre. (76-77)

éveil : Deuxième avertissement de Jim. (80)

15. conscience: Alain est «englué dans ses mots». (82)

fuite: «Je me résignerais à une partie de solitaire pour ne pas penser. " (83)

éveil: $\quad$ Alain évoque l'avertissement de Jim. (84)

16. conscience: Alain analyse la réaction de Madeleine. (84)

fuite: "Les mots de Jim, je n'y crois plus tout à coup. »

II est intéressant de noter, cette fois-ci, un rythme du complexe au simple. Plus on avance dans le récit, moins la fréquence devient obsédante, à un point tel qu'il n'en est plus question dans la seconde partie. On passe donc d'une fréquence 8 (les avertissements de Kouri), à une fréquence 5 (Alain songeant à Kouri), à une fréquence 3 (les avertissements de Jim), puis à l'absence de tout phénomène de fréquence.

\subsection{Forme et signification du discours temporel}

La forme du discours temporel de Poussière, schématisée (quoi qu'en pense Butor), peut prendre cette configuration (voir p. suivante):

Les analepses présentent, on le voit, une forme qui va du complexe au simple. Cette constatation revêt, quant à la signification du récit, une importance considérable. Poussière est, en son début, le récit d'une mémoire. Ce phénomène est, on le sait, une réalité formelle mais, au surplus, il est une donnée signifiante: la rupture s'accompagne d'une remontée dans l'être. Au début, Alain ne s'interrogeait pas, il vivait un «demisommeil " où Madeleine et lui s'étaient étreints "à l'aveuglette"; tout avait été fait avec passivité. Et voilà qu'il faut, à cause de Kouri, remonter le courant, récupérer des antécédents fautifs parce qu'avant la faute la conscience n'éclairait rien. Rétrospectivement, Alain a «un droit de reprise sur l'univers" où il tente une mise en ordre des choses. La vie, inaugurée par la conscience dont Kouri se fait le catalyseur, devient un filet à tisser où il faut "relier entre elles ces images d'un passé tout neuf " ( $P$., p. 38). Le manque, la faute est à l'origine du récit, et la mémoire a pour tâche de rendre à sa manière l'univers cohérent: plus qu'une simple technique du récit, la remontée dans le temps est la première démarche de l'homme non situé. S'il y a échec dans Poussière, ce n'est pas à la fin, mais au début: Alain découvre que son passé, parce que non conscientisé, contamine son présent, et il lui faut brûler ces souvenirs afin de les empêcher de perturber ce présent. Ainsi, dès que le temps apparaît comme mémoire, l'échec est en un certain sens dépassé. 


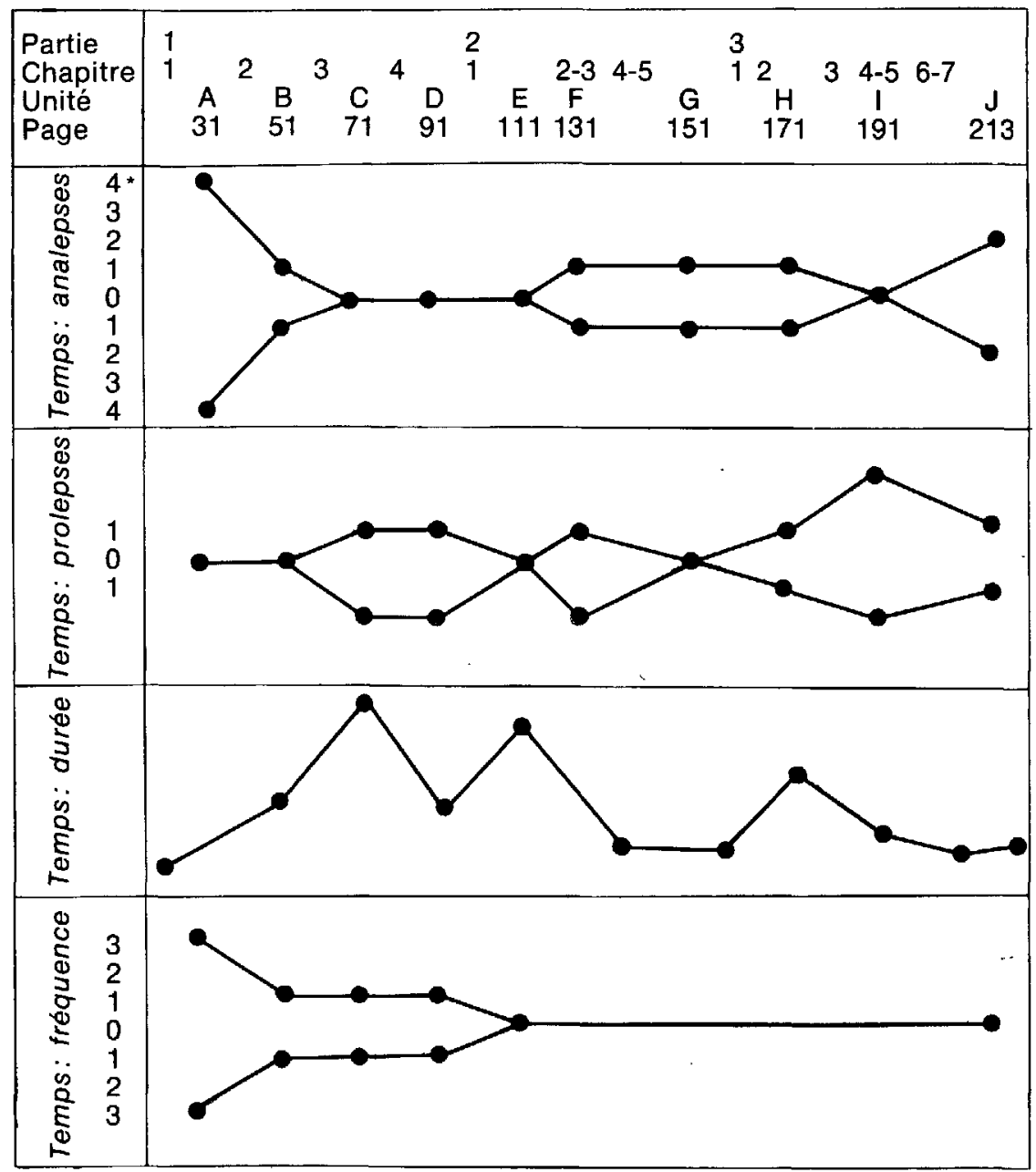

* Les chiffres verticaux, dans le tableau, présentent une graduation qui rendra compte du nombre de phénomènes temporels relevés (sauf pour la durée, dont le tableau reproduit celui de la page 219). Les chiffres verticaux indicateurs sont $31,51,71$, etc., qui proposent une division du roman en tranches de 20 pages (le roman débute à la page 11). Il s'ensuit, dans le cas des analepses par exemple, que la tranche des pages 11-31, dite $A$, contient 8 cas, traduits dans le schéma par un double trait qui s'amorce au niveau des chiffres 4 et 4 .

Souvenons-nous maintenant des prolepses: huit phénomènes, présentant un mouvement du simple au complexe. Le récit commence par une rupture, et s'achèvera par une réconciliation. La fin revêt chez Alain la forme d'une décision: "Je resterai." Cette décision marque chez lui le dépassement de l'échec initial, et ce dépassement nous le retrouvons dans le contenu progressif des huit prolepses: 
1. fatalité

2. fatalité

3. fatalité

4. prémonition (fatalité)
5. décision

6. fatalité

7. espoir

8. décision

Le contenu des prolepses décrit un passage du subi au décidé, comme s'il y avait une prise en charge progressive par Alain de son propre destin, après l'apprentissage initial de la vérité.

La durée du récit corrobore la même forme et la même signification. Le rythme est lent, rapide puis lent. D'abord lent: au début, l'échec s'installe et le récit fouille la plaie initiale. Puis rapide: les événements se bousculent, l'histoire se charge d'événements. Puis lent: le récit s'arrête pour contempler sa victoire, l'échec est dépassé.

La fréquence épouse une configuration complexe-simple. Cela n'a rien d'étonnant, puisque le récit fouille l'échec initial non seulement par la lenteur de son rythme, mais aussi par la répétition obsédante de la cause primordiale du déchirement: l'avertissement de Kouri. L'échec est réitéré, pour que le feu inaugural ne s'éteigne pas.

\subsection{FORME ET SIGNIFICATION DU DISCOURS IMMÉDIAT}

Nous abordons maintenant la seconde instance de notre analyse, le discours immédiat, selon notre démarche maintenant connue: fonctionnement puis forme et signification.

\subsection{Le fonctionnement du discours immédiat}

Le discours immédiat, forme mimétique par excellence, désigne ce que la critique a toujours baptisé monologue intérieur: pourtant, le premier terme sera préféré au deuxième, pour deux raisons. L'une, pour maintenir une certaine continuité dans la définition des termes (discours direct, indirect, immédiat); l'autre, pour éviter un certain psychologisme plus embarrassant qu'éclairant 20 .

Mais, encore une fois, nommer l'objet d'analyse n'a rien à voir avec sa description. Une rose sentirait aussi bon même si elle portait un autre nom, disait Shakespeare; et ainsi, monologue intérieur ou discours immédiat, la difficulté reste posée: comment le repérer dans le texte?

Dans un article récent, Philippe Hamon a soulevé certaines difficultés au sujet de la description, qui sont parentes avec celles rencontrées ici quant au discours immédiat.

La difficulté rencontrée par Hamon, parlant de la description, peut grosso modo se résumer à ce qui suit: comment définir une description 
"comme unité spécifique, sur des critères formels et/ou fonctionnels précis $^{21}$ "? En conséquence, Hamon suggère un problème à trois termes:

a) la façon dont la description, par des signes démarcatifs, s'insère dans le texte;

b) la façon dont elle fonctionne intérieurement;

c) son rôle dans la narration.

Cette recherche de critères d'insertion et de fonctionnement peut guider notre activité dans notre quête du discours immédiat. Un lecteur sent bien la présence d'un niveau, d'une instance différente dans l'acte de sa lecture; mais c'est au moment de définir cette impression et d'en décrire la cause qu'il achoppe. Cela revient à dire que l'emploi du présent et $\mathrm{du}$ "je» ne suffit pas à définir le discours immédiat. C'est plus loin qu'il nous faut aller; et, à ce point, partons d'un exemple qui serait, hypothétiquement, un segment de discours immédiat.

Je n'arrive pas à m'expliquer mon émoi, la chaleur intérieure qui m'a bouleversé. Parce que Kouri me révélait une part de la vie de Madeleine que je ne connaissais pas? Peut-être. Comme s'il avait levé le rideau et $m$ 'avait montré derrière une vitre un être dont j'aurais ignoré complètement l'identité et qui eût été ma femme. Madeleine m'échappait par plusieurs points. C'était cela mon émotion. Je ne la soupçonnais quand même pas. Et la soupçonner de quoi ? Il ne faut surtout pas que je me laisse séduire par le jeu des images. Imaginer Madeleine dans le restaurant du Syrien souriant à quelqu'un qui lui parle. Quand bien même cela serait, il y aurait mille interprétations possibles en plus de celle que ma chair appelle. Non. Je n'ai ni le goût, ni le temps de ces sortes de jeux. (P., p. 13)

On peut découper facilement ce segment narratif, dans la mesure où ce découpage rendra compte de l'insertion et du fonctionnement du discours:

I: un personnage qui a joué (et qui joue) un rôle d'influenceur, et qui, de là, entraîne une réflexion intérieure (ici : Kouri);

$P$ : un personnage qui prend en charge le discours immédiat (ici: Alain);

$\mathrm{TH}$ : un thème introducteur (qui est ici celui de l'ignorance);

$+$

s: des signes linguistiques de ce thème (qui sont ici le point d'interrogation, le conditionnel, les locutions modalisantes);

$F$ : une activité de $\mathbf{P}$ (en opposition avec $\mathrm{TH}$, et qui sera la tentative d'expliquer);

C: une clôture du discours («Je n'ai ni le goût, ni le temps de ces sortes de jeux. ").

Le formule de ce segment, considéré hypothétiquement comme discours immédiat, serait:

$$
\text { D.I. }=I+P+(T h+s)+F+C
$$

Prenons maintenant un autre exemple:

Elle [I, Madeleine] libère en moi $[\mathrm{P}]$ un torrent de pitié dont je désire l'abreuver [F]. Comme si elle me passait sa souffrance [TH] tout 


\begin{tabular}{|c|c|c|c|c|}
\hline $\begin{array}{r}\text { Discours } \mathrm{n}^{\circ} \text { : } \\
1 . \\
2 . \\
3 . \\
4 . \\
5 . \\
6 . \\
7 . \\
8 . \\
9 . \\
10 .\end{array}$ & 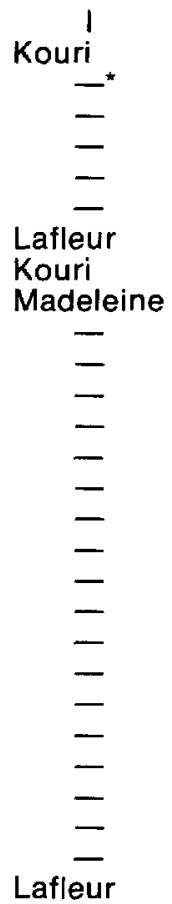 & $\begin{array}{l}P \\
\text { Alain } \\
= \\
= \\
= \\
= \\
= \\
= \\
= \\
= \\
= \\
= \\
= \\
= \\
= \\
= \\
= \\
=\end{array}$ & 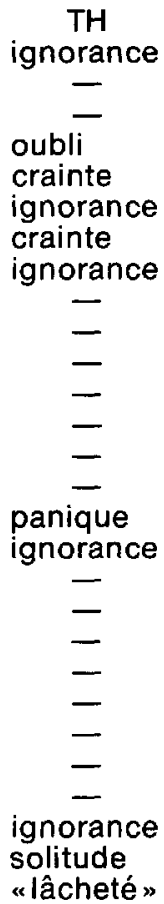 & $\begin{array}{l}\text { exclamations } \\
\text { négations } \\
\text { négations } \\
\text { négations } \\
\text { négations } \\
\text { comme si } \\
\text { loc. modalisantes } \\
\text { négations } \\
\text { loc. modalisantes } \\
\text { exclamations } \\
\text { interrogations } \\
= \\
= \\
= \\
\text { négations } \\
- \\
\text { interrogations } \\
\text { loc. modalisantes } \\
\text { conditionnel } \\
\text { interrogations }\end{array}$ \\
\hline
\end{tabular}

\begin{tabular}{|c|c|}
\hline $\begin{array}{c}F \\
\text { explication }\end{array}$ & fuite ${ }^{C}$ \\
\hline 二 & = \\
\hline $\begin{array}{l}\text { abandon } \\
\text { décision } \\
\text { explication }\end{array}$ & $\begin{array}{l}\text { fuite } \\
\text { fuite }\end{array}$ \\
\hline $\begin{array}{l}\text { explication } \\
\text { justification } \\
\text { explication }\end{array}$ & fuite \\
\hline explication & acceptatic \\
\hline $\begin{array}{l}\text { abandon } \\
\text { explication }\end{array}$ & fuite $=$ \\
\hline - & fuite \\
\hline- & fuite \\
\hline - & - \\
\hline- & décision \\
\hline- & - \\
\hline - & décision \\
\hline $\begin{array}{l}\text { imagination } \\
\text { explication }\end{array}$ & $\begin{array}{l}\text { fuite } \\
\text { décision }\end{array}$ \\
\hline
\end{tabular}

* Le trait indique une répétition. 
entière et que j'acceptasse de m'en charger. L'émotion me fait trembler. Cette femme pantelante $n$ est plus la mienne, je ne me reconnais plus aucun droit sur elle $[\mathrm{TH}]$. [...] Je ne $[+\mathrm{s}]$ la rejoindrai jamais, jamais $[+s]$. Nous ne $[+s]$ pouvons nous acharner à rapprocher nos deux lignes parallèles. [...] Je ne suis plus son mari, je suis son allié contre l'absurde cruauté [F]. [...] Tout devient limpide pour moi maintenant. Je souffrirai encore par Madeleine, je le sais, mais je ne m'indignerai plus $[C]$, je ne l'en accuserai plus $[C]$. (P., p. 152)

Le fonctionnement de ce segment de discours immédiat serait:

I: Madeleine;

P: Alain:

$\mathrm{TH}$ : Madeleine étrangère et sa souffrance ( $+\mathrm{s}$ : négations);

F: être son allié, l'abreuver de pitié;

C: segment proleptique décisionnel.

Le texte de Poussière sur la ville comporte en tout vingt-huit segments de discours immédiat. Une taxonomie, telle que celle établie plus haut mais englobant tous les discours immédiats, peut être utile pour comprendre l'allure générale du texte (voir tableau p. précédente).

\subsection{Forme et signification du discours immédiat}

Reprenant les mêmes fondements, nous pouvons compléter le tableau des formes temporelles en y ajoutant maintenant celui de la forme du discours immédiat:

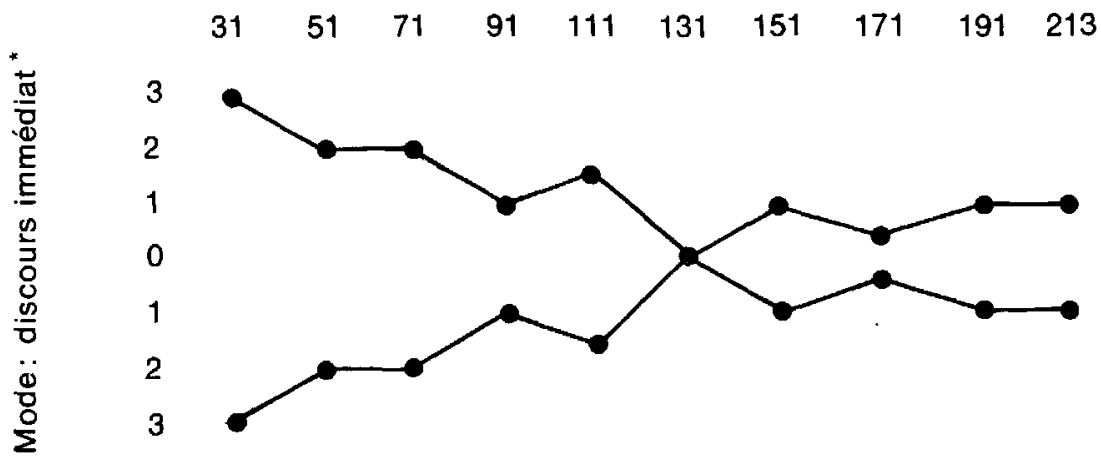

* Ce tableau s'organise de manière identique à celui de la page 223 (et il peut donc lui être superposé): tranches de 20 pages, et nombre de segments de discours immédiat indiqué pour chacune des tranches par les chiffres verticaux. Exemple: la tranche A (pages 11-31), contient 6 cas de discours immédiat.

La signification du discours immédiat semble être riche d'enseignements. La situation initiale du récit est une situation de rupture et d'interrogations. Le «je» et sa raison quêtent l'intelligibilité d'un univers en 
décomposition, l'homme veut remanier son monde dans son cerveau, et cette recomposition relève de la pensée approximative dont la manifestation est le monologue intérieur.

«Le stream concrétise une dialectique sur laquelle reposent les formes les plus originales de l'art du xxe siècle: I'homme aspire d'autant plus à l'unité et à la profondeur qu'il se sent fragmenté, nivelé et aliéné tout ensemble par la vie moderne ${ }^{22}$." Le discours immédiat n'est pas seulement une forme, il représente aussi un mode d'être, une médiation avec le monde. Devant l'éparpillement d'une vie, le seul recours reste la parole intérieure approximative: elle tente, par des mouvements de va-etvient perpétuels, de filer en ligne droite. Ce type de discours recompose un monde à la manière d'une mosaïque: la parole est à l'image de son référent, fragmentée. II n'est pas étonnant que le discours immédiat se caractérise, entre autres, par l'absence de destinataire: en rupture, le “je" se proclame centre du monde, emprisonné dans son discours autarcique. II n'est pas étonnant, également, que ce type de langage se nourrisse goulûment de locutions modalisantes (dont nous avons déjà parlé): l'intensité de la vérité se mesure à la quantité des approximations et des hésitations. Le fonctionnement du discours immédiat nous a bien révélé ce caractère d'impuissance du langage rationnel: il doit son origine à l'ignorance surtout (cf. TH), progresse par le détour des peut-être et des si, et se clôt la plupart du temps par un retour à l'ignorance (cf. C).

Le contenu du discours immédiat, dévoilé dans sa phase $\mathrm{C}$ (Clôture), nous fait passer de l'échec à la victoire (plus précisément de la fuite à la décision). Cette constatation amène de l'eau à notre moulin quant à la signification ultime de Poussière: le récit d'une victoire.

\subsection{CONCLUSION}

Nous espérons avoir démontré, en dépit de la brièveté de notre travail, après l'analyse du fonctionnement du temps et du discours immédiat, que la forme de Poussière sur la ville, complexe, simple, puis complexe, porte en ses extrémités l'échec initial et la victoire finale. Avec plus de temps, cette hypothèse (qui n'en est plus une, nous l'espérons) aurait pu être étayée par l'analyse de la focalisation, de la voix, de l'espace et de la grammaire du récit.

Afin de donner une vision totale des propos antérieurs, nous proposons le tableau suivant: 


\begin{tabular}{|c|c|c|c|c|}
\hline & & $\begin{array}{l}\text { Début du } \\
\text { récit: } \\
\text { Pages } 11-60 \\
\text { environ }\end{array}$ & $\begin{array}{l}\text { Centre } d u \\
\text { récit }\end{array}$ & $\begin{array}{c}\text { Fin du récit: } \\
\text { Pages 198-213 } \\
\text { environ }\end{array}$ \\
\hline Analepses & $\begin{array}{l}\text { Forme } \\
\text { Signification }\end{array}$ & $\begin{array}{l}\text { Complexe } \\
\text { Brisure initia- } \\
\text { le;conscience } \\
\text { d'une sépara- } \\
\text { tion: échec }\end{array}$ & Simple & Simple \\
\hline Prolepses & $\begin{array}{l}\text { Forme } \\
\text { Signification }\end{array}$ & Simple & Simple & $\begin{array}{l}\text { Complexe } \\
\text { Décision } \\
\text { finale: } \\
\text { Réparation } \\
\text { Victoire }\end{array}$ \\
\hline Durée & $\begin{array}{l}\text { Forme } \\
\text { Signification }\end{array}$ & $\begin{array}{l}\text { Lente } \\
\text { Contempla- } \\
\text { tion de la } \\
\text { déchirure } \\
\text { initiale }\end{array}$ & Vite & $\begin{array}{l}\text { Lente } \\
\text { Médiation de la } \\
\text { victoire finale }\end{array}$ \\
\hline Fréquence & $\begin{array}{l}\text { Forme } \\
\text { Signification }\end{array}$ & $\begin{array}{l}\text { Complexe } \\
\text { Réitération de } \\
\text { la déchirure } \\
\text { initiale }\end{array}$ & Simple & Simple \\
\hline $\begin{array}{l}\text { Discours } \\
\text { immédiat }\end{array}$ & $\begin{array}{l}\text { Forme } \\
\text { Signification }\end{array}$ & $\begin{array}{l}\text { Complexe } \\
\text { Contenu: fuite }\end{array}$ & Simple & $\begin{array}{l}\text { Complexe } \\
\text { Contenu: décision }\end{array}$ \\
\hline
\end{tabular}

Poussière sur la ville apparaît ainsi comme le récit d'une victoire, par le biais du passage du subi au décidé dans la perspective d'Alain, dans la mesure où cette constatation s'est fondée sur la forme du discours narratif.

II serait intéressant d'élargir cette perspective de «la déchirure initiale" et de "la victoire finale " à toute la littérature, afin de voir si, en réalité, l'écriture ne se nourrit pas du vide qu'elle tente de combler. L'amour, dit Platon, manque de la beauté et de la bonté qu'il désire; l'écriture aussi, manque peut-être de beauté et de bonté et, à ce titre, la clôture du texte marquerait toujours la réussite ou l'échec dans cet effort pour combler le vide initial.

Nous espérons avoir démontré que la forme d'un récit littéraire, telle qu'elle a pu être mise à jour par les techniques d'analyse que nous avons à notre disposition, ne résulte pas d'une dissémination aléatoire des éléments pertinents du texte. Sans doute la théorie de l'information, qui se propose de mesurer la complexité des éléments d'un message, pourrait fournir un instrument de travail plus rigoureux pour une mesure de la forme du texte et de l'information qui résulte de sa complexité. C'est dans cette direction que nous proposons de nouvelles recherches. 
1. Le présent article propose une partie remaniée de notre thèse de M.A. présentée à l'Université de Sherbrooke en juin 1974. La thèse elle-même étudie la forme du discours narratif de Poussière dans son ensemble (temps, mode, voix, espace) et cherche ensuite la signification mythique de cette même forme.

2. Voir I. Rousset, Forme et signification, Paris, Jose Corti.

3. Michel Butor, Essais sur le roman, Paris, Gallimard, "ldées", p. 114.

4. Gérard Genette, Figures III, Paris, Seuil, "Poétique"; voir ordre, fréquence, durée, p. 77-182.

5. Claude Bremond, "la Logique des possibles narratifs", dans Communications, $n^{\circ} 8,1966$, p. 76.

6. Id., Logique du récit, Paris, Seuil, “Poétique», p. 131-134.

7. Id., «la Logique des possibles narratifs», p. 62.

8. Émile Benveniste, Problèmes de linguistique générale, p. 246.

9. Nous rejetons l'interprétation du temps en termes de modes verbaux que fait Van Laere, dans Une lecture du temps dans "La Nouvelle Héloïse", pour donner raison à Genette: le récit, à strictement parler, est à l'indicatif (Figures III, p. 183).

10. C'est-a-dire «un assemblage normalisé et routinier de signes du niveau inférieur-» (Abraham Moles, Art et ordinateur, Casterman, 1971, p. 26) Cette hiérarchie est possible grâce au statut d'intégrant du signe linguistique, et en vertu du fait que nous considérons le récit littéraire comme phénomène sémiologique.

11. Rappelons que pour Genette, TN = temps du discours narratif (nécessairement linéaire) et $\mathrm{TH}=$ temps de l'histoire identifiée chronologiquement.

12. II est évident que le choix des événements est arbitraire de même que leur numérotation; tout cela n'a qu'une valeur de repérage.

13. Désormais les références à Poussière sur la ville seront désignées dans le texte par $P, \mathrm{p} \ldots$

14. Gérard Genette, op. cit., p. 96.

15. Ibid., p. 98.

16. Ibid., p. 88.

17. Ibid., p. 123.

18. Ibid., p. 124.

19. C'est-a-dire que ce qui se produit $n$ fois dans le récit n'arrive qu' une fois dans I'histoire.

20. Ajoutons que ce type de discours est aussi «immédiat", parce qu'il est «émancipé de tout patronage narratif $"$ : voir Figures III, p. 193.

21. Philippe Hamon. "Qu'est-ce qu'une description?", dans Poétique, $n^{\circ} 12$.

22. Michel Zeraffa, la Révolution romanesque, Paris, UGE, p. 152-153. 